\title{
Editorial
}

\section{Control, Analysis, and Modeling of Vehicular Systems}

\author{
Ilse Cervantes, ${ }^{1}$ Sheldon S. Williamson, ${ }^{2}$ Ali Davoudi, ${ }^{3}$ and Luis Alvarez-Icaza ${ }^{4}$ \\ ${ }^{1}$ Institute for Scientific and Technological Research of San Luis Potosi (IPICyT), Camino a la Presa San Jose 2055, \\ Col. Lomas 4ta, 78216 San Luis Potosi, SLP, Mexico \\ ${ }^{2}$ Department of Electrical and Computer Engineering, Concordia University, Montreal, QC, Canada H4G $2 \mathrm{M1}$ \\ ${ }^{3}$ Electrical Engineering Department, University of Texas at Arlington, Arlington, TX 76019, USA \\ ${ }^{4}$ Institute of Engineering, Universidad Nacional Autonoma de Mexico, 04510 Mexico City, DF, Mexico \\ Correspondence should be addressed to Ilse Cervantes; ilse@ipicyt.edu.mx
}

Received 29 April 2014; Accepted 29 April 2014; Published 1 July 2014

Copyright (C) 2014 Ilse Cervantes et al. This is an open access article distributed under the Creative Commons Attribution License, which permits unrestricted use, distribution, and reproduction in any medium, provided the original work is properly cited.

\section{Introduction}

Automation has played a key role in the development of new technologies and in the maturation and improvement of existing ones. Automatic control launched its development with electronic devices since the mid-twentieth century, but many of the key mathematical tools for dynamic analysis and control synthesis were developed much earlier. It was necessary to join the mathematics with the electronics and other related areas such as simulation and modeling to raise this promising, now essential area of knowledge.

The number of automatic processes applied to vehicles and transportation systems has increased dramatically in recent years due to the growing urge of environmentalfriendly and autonomous transportation. As technology advances, more complicated mathematical problems have to be solved in order to satisfy the increasing astringent expectations on safety, reliability, and performance of vehicular systems.

Our intention with this special issue was to provide an opportunity for researchers to share their latest theoretical and technological achievements in control, modeling, and analysis of vehicular systems and to become a forum to contribute, from a mathematical point of view, to the solution of some of the challenges that the development of new trends of transportation has arisen.

This special issue focuses on the state-of-the-art research and development in the areas of control and analysis of transportation systems as well as in current trends as, for example, transportation electrification, vehicular communication systems, and driver assistance applications. For this special issue, we received many submissions and after conducting a rigorous review process, we have accepted only 11 high-quality papers.

The articles in this special issue are classified into three topics: those related to stabilization and control of vehicular systems, which concern either with control synthesis and stabilization capabilities of controllers of vehicular systems or dealing with vehicular related applications, for example, a haptic steering by wire system based on high gain GPI observers, the application of a dynamic surface control to lateral vehicle control, or a vehicular wireless communication problem solved from a control point of view.

The second topic in this issue regards the application of analysis or numerical methods to vehicular problems, among these we can cite a sound signal processing algorithm for vehicular-type recognition or an approximation to the vehicle routing problem using an ant colony algorithm. Finally, the third topic regards transportation electrification problems, which concern modeling or control problems related to the electrification of vehicles, for example, the development of control strategies for energy management of an hybrid electric bus.

In the rest of this document we give a short description of the papers in this special issue. We sincerely hope that this special issue serves as a reference for research in the area 
of applied mathematics to transportation and provides an incentive to attract talents which contribute to this fascinating area.

\section{Stability and Control of Vehicular or Vehicular-Related Systems}

One of the topics covered in this special issue is related to the control and stabilization of vehicular systems, including theoretical analysis of stabilization and its use in the solution of vehicle related problems. There are six papers related to this topic. The paper entitled "Analysis and optimization of resource control in high-speed railway wireless networks" by S. $\mathrm{Xu}$ et al. studies the joint optimal design of admission control and resource allocation for multimedia services delivery in high-speed railway wireless networks. Its main contribution is to propose a stochastic optimization framework for transmission, which focuses on the joint admission control and resource allocation problem under an average power constraint. Using virtual queues, the joint admission control and resource allocation problem, originally formulated as a stochastic optimization problem, is transformed into a queue stability problem.

On the other hand, the transformed problem is decomposed into three subproblems: admission control, utility maximization, and resource allocation. The use of the driftplus-penalty approach transforms the resource allocation problem into a single variable problem, which is used to develop a distributed dynamic packet loading algorithm with guaranteed global optimality. To the best of our knowledge, it is the first time that the Lyapunov optimization theory is extended into the HSR wireless networks.

Another paper of this topic is "Stability of gain scheduling control for aircraft with highly nonlinear behavior" by $\mathrm{F}$. Mendez-Vergara et al. The main goal of this work is to study the stability properties of an aircraft with nonlinear behavior. The problem of body stabilization and attitude control is solved using a piecewise linear output feedback and asymptotical stability of the task coordinates origin and safety of the operation in the entire flight envelope. The paper departs from a general description of the aircraft that uses piecewise nonlinear models. This paper has two main contributions: firstly, to deal with nonlinearities of the description of the aircraft from a unified point of view, in which the effects of the parametric uncertainty, structural uncertainty, and some control failures can be studied with a single description and secondly, to propose relaxed switching conditions for stability that can depend either on time, output, or the entire state and they can be applied using a supervisory structure which evaluates the satisfaction of the stability conditions. The result proposed by the authors is interesting since even if the behavior of the aircraft is highly nonlinear, the controller can be synthesized using a piecewise linear description of the system in a given operation points, and the performance of the system can be set using a trade-off between the size of integral terms and the size of the operating domain.

On the other hand, in " $A$ vehicle haptic steering by wire system based on high gain GPI observers" by A. Rodriguez
Angeles et al., a vehicle steering by wire (SBW) haptic system based on high gain generalized proportional integral (GPI) observers is introduced. A steer-by-wire system is a modular electric power assisted steering device with an absence of steering column, steering shaft, or mechanical connection between the steering wheel and the road wheel. SBW requires control actions to steering tracking and the observers in this paper are used for the estimation of dynamic perturbations that are present at the tire and steering wheel. The estimated dynamic effects and position tracking errors are feedback yielding a master-slave haptic system with bilateral communication. The main contribution of this paper is to propose a steering by wire (SBW) haptic system that requires the nonlinear system input gain of the system and that uses only encoders to feedback purposes. The tracking error between the desired angle settled by the human operator, and the tire orientation angle can be made arbitrarily small by a proper gain selection. Using experiments, the authors show that the controller is robust to uncertainty on the input gain and that it is able to cancel dynamic perturbation effects such as friction and aligning forces on the tire.

Along the line of developing controllers for advanced driver assistance systems, there is the paper "Robust switched predictive braking control for rollover prevention in wheeled vehicles" by M. R. Licea and I. Cervantes. This paper proposes a differential braking rollover mitigation strategy for wheeled vehicles. Using a polytopic description of the vehicle that includes suspension effects and translational and rotational dynamics of the vehicle, the authors propose a velocitydependent domain partition and a predictive action for preventing rollover. To predict the behavior of the vehicle, the authors use a linear model and a low-order model of the driver behavior. The proposed methodology, which constitutes the main contribution of the paper, is adaptable via switching actions, interfering with driver's acceleration/braking actions only when a risk of rollover is foreseen. The proof of stability of the scheme is performed in two steps; firstly, stability conditions for the hybrid closed-loop system are derived with respect to a switching surface that depends on norm of system states. Secondly, the authors prove that the switching surface can be time varying, leading to a more relaxed switching conditions by the use of prediction.

An interesting paper that performs an extension of known control methodologies is the paper "Dynamic surface control and its application to lateral vehicle control" by B. Song et al. This paper extends the design and analysis methodology of dynamic surface control (DSC) for a class of nonlinear systems where the nonlinear functions are included as forcing terms. In many applications such as rotational mechanical systems, sinusoidal functions are included in the equation of motions; however, when the sinusoidal function is used as a forcing term for DSC, the stability analysis is complicated by highly nonlinear functions resulting from the low-pass filter dynamics. With a modification of input variables to the filter dynamics, the burden of mathematical analysis can be reduced and stability conditions in linear matrix inequality are formed to guarantee the quadratic stability. The illustration of this proposed strategy is applied to lateral vehicle control for 
forward automated driving and backward parallel parking at a low speed.

The last paper of this topic is " $\mathrm{H}^{\infty}$ controller design for asynchronous multirate sampled-data systems" by X. Sun and W. Mao. Multirate sampled-data systems are the product of different sampling rates of the signals of interest. In the implementation of energy management strategies of hybrid electrical vehicles, a hierarchical controller is designed to perform the power split among the sources, but as the system becomes large and complex, an unavoidable situation of sensors and control inputs evolving at different sampling rates may be present. In these cases, multirate sampleddata systems are better choices for modeling in order to improve the closed-loop performance. This paper proposes an observer-based $\mathrm{H}^{\infty}$ controller which guarantees that the $\mathrm{H}^{\infty}$ norm of the closed system is less than a given attenuation level. To improve the performance further, the exogenous signals sampled at different rates are lifted to an appropriate signal rate, while the endogenous signals are not lifted to avoid a causal constraint. The controller is obtained by solving a matrix inequality.

\section{Analysis and Numerical Methods Applied to Vehicular Problems}

Vehicle-type recognition, which can be used in intrusion detection, transportation, and border monitoring among others, is a demanding application for wireless sensor networks. Traditionally, sensor nodes detect and recognize vehicles from their acoustic or seismic signals using wavelet based or spectral feature extraction methods. However, such methods are demanding in computational power and energy and are difficult to implement on low cost sensor nodes with limited resources. In "Vehicular type recognition in sensor networks using improved time encoded signal processing algorithm" by Y. Wang et al., the use of time encoded (acoustic) signal processing algorithm for vehicle recognition is investigated. An improved time encoded signal processing is proposed as the feature extraction method according to the characteristics of the vehicle sound signal. Recognition procedure is accomplished using a support vector machine and a $k$ nearest neighbor classifier. The authors validate their proposed methodology using experimental results and illustrate that the ITESP features have a better performance compared with the conventional TESP methodologies.

On the other hand, a paper that looks into the path planning of an emergency rescue vehicle in an interesting way is "An improved ant colony algorithm and its application in vehicle routing problem" by $\mathrm{M}$. Huang and $\mathrm{P}$. Ding. The vehicle routing problem is defined as a transportation and a distribution problem with the constrained of vehicle load, travel time, or distance. In this paper, an ant colony optimization algorithm is applied to the optimal path planning of an emergency rescue vehicle. With respect to existing methodologies, the proposed algorithm uses a new transition probability function adding the angle factor function and visibility function, while setting penalty function in a new pheromone updating model to improve the accuracy of the route searching. The results presented by the authors show that the proposed method is better than the traditional ant colony algorithm for vehicle routing path planning and that the method can plan a more rational rescue path focused on a real traffic situation.

Robust object detection and tracking is a major technological issue that has to be solved for vehicle automation, in particular for automated steering and acceleration. In "Invariant Hough random ferns for object detection and tracking" by Y. Lin et al., a robust object detection methodology under changes in object appearance, scale, partial occlusions, and pose variation is proposed. The methodology incorporates rotation and scale invariance into a local feature description, random ferns classifier training, and Hough voting stages. The contributions of the authors are three: the rotation invariant local binary feature based on polar coordinates is shown to be invariant to image rotation; the combined use of Hough transform with random ferns classifier provides object detection regardless of partial occlusions and nonrigid deformations; and finally, a refined top-down segmentation algorithm based on a clustering scheme is presented. The authors show using experimental results that both object segmentation and long-term object tracking are accurate and robust in a variety of complex scenarios.

\section{Transportation Electrification}

J. Wang et al. in the paper "The development and verification of a novel ECMS of hybrid electric bus" proposed an adaptable equivalent consumption minimization strategy for a hybrid electric bus, which coordinates the relationship between the gear-shifting and motor assistance. The strategy also performs drive cycle recognition to update the charge and discharge coefficients in order to achieve better fuel economy in each drive cycle. Hardware-in-the-loop tests in a seriesparallel hybrid electric bus show that the proposed method can achieve significant fuel economy with respect to other existing methodologies and provide new design ideas for the gear-shifting rules. Finally, in "Sample-data modeling of a zero voltage transition DC-DC converter for on-board battery charger in $E V^{\prime \prime}$ by T. R. Granados-Luna et al., a sampledata model for a battery charger is proposed. The authors analyze the idealized waveforms of the converter to perform a dynamical analysis or the system; then a phase control strategy is modelled to obtain a large-signal model of the converter. Using this model, a half-cycle, sample-data linear model is obtained, which helps to provide the small-signal transfer functions of the converter.

\section{Acknowledgments}

We are grateful to our reviewers who dedicated their time in reviewing the submitted papers and provided valuable suggestions to the authors.

Ilse Cervantes

Sheldon S. Williamson

Ali Davoudi

Luis Alvarez-Icaza 


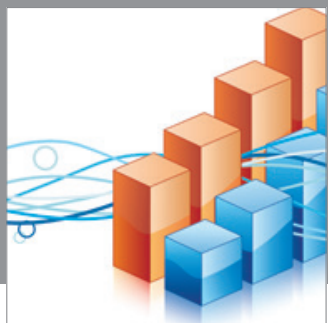

Advances in

Operations Research

mansans

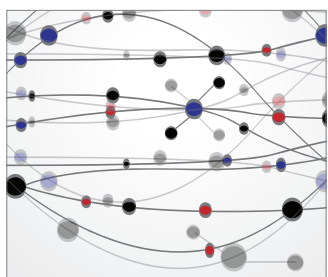

The Scientific World Journal
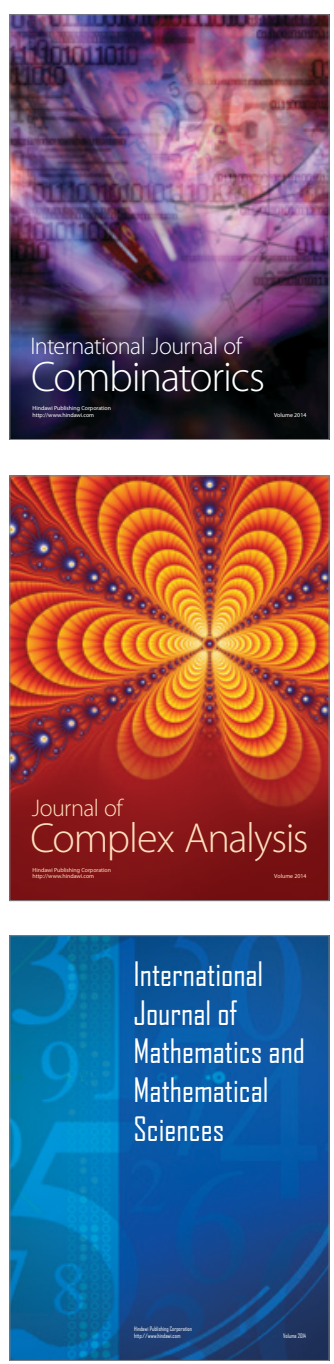
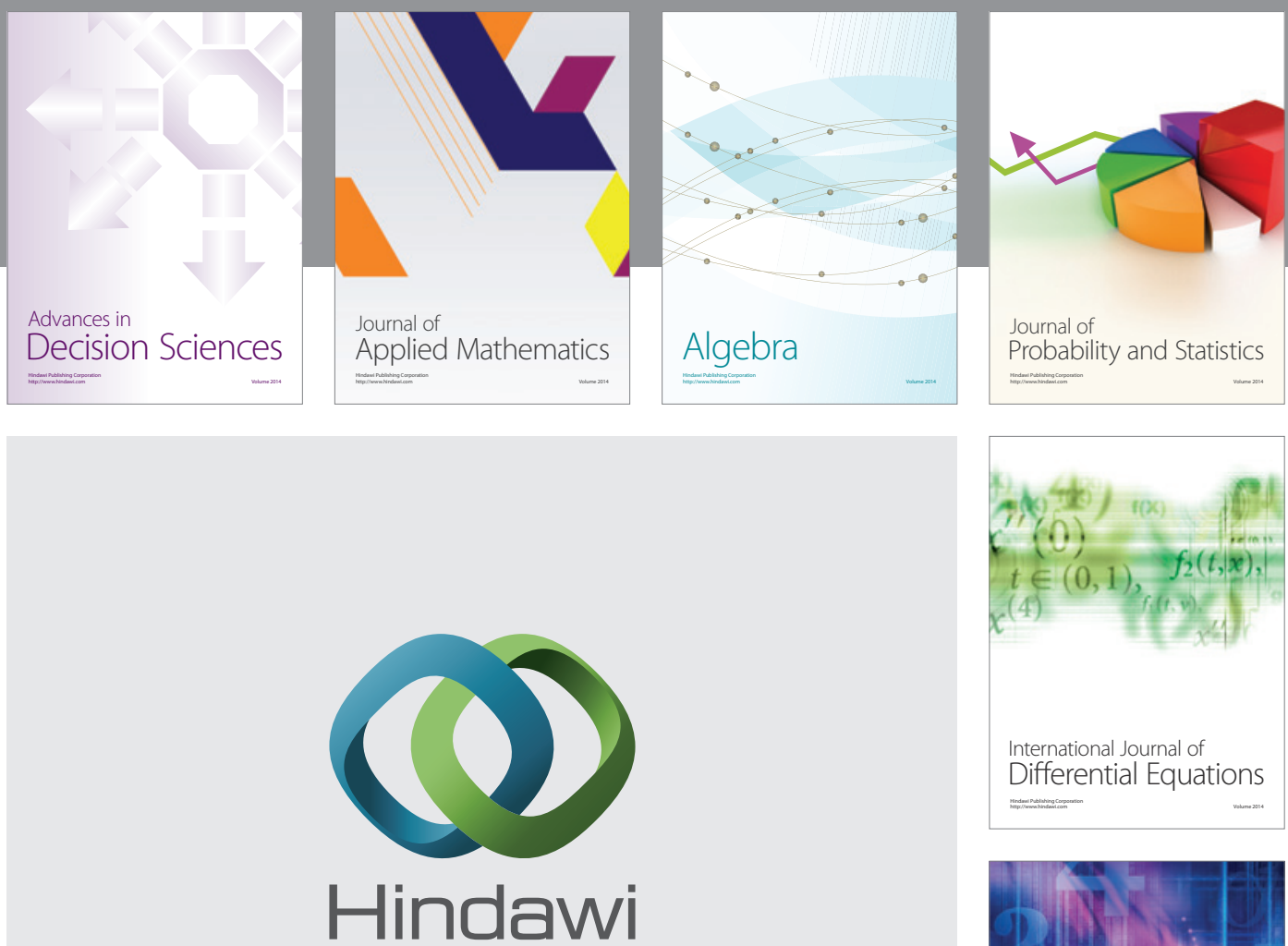

Submit your manuscripts at http://www.hindawi.com
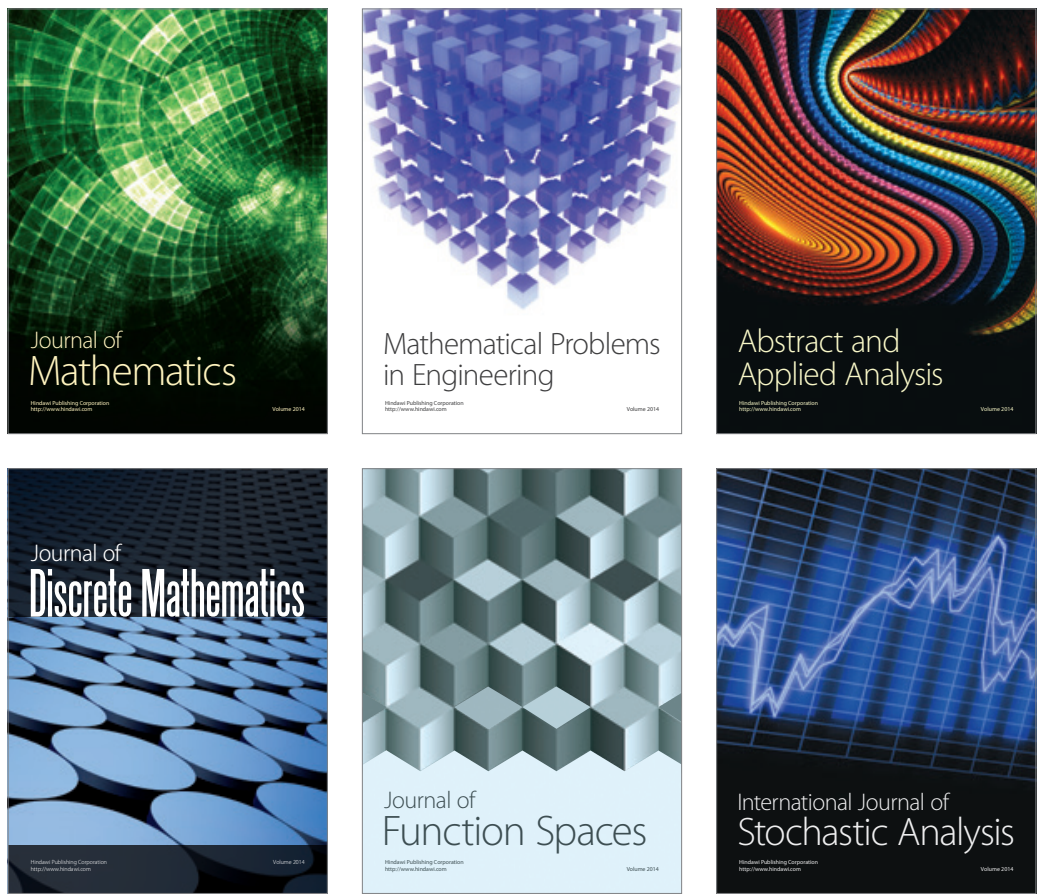

Journal of

Function Spaces

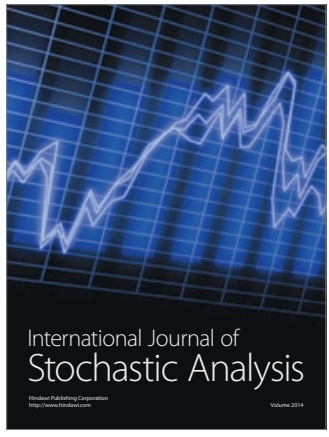

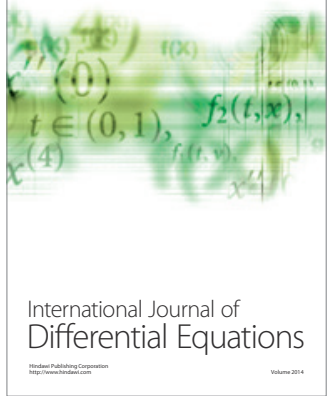
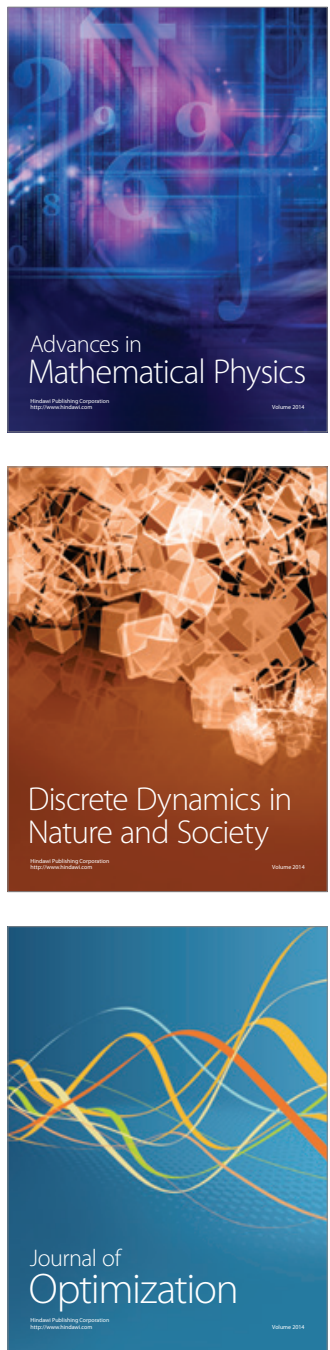